\section{Scattering sources}

\section{R.A. Cowley}

Theory of Neutron Scattering from

Condensed Matter, Vols 1 and 2.

By Stephen W. Lovesey.

Clarendon: 1984. Vol. 1 pp.329. Vol. 2

pp.344. £38, \$59 each volume.

NEUTRON scattering now plays an essential role in our understanding of many properties of condensed matter, and is used in the study of atomic structures, interatomic forces, magnetism, liquids and polymers. Stephen Lovesey has made significant contributions on the theoretical side of the subject. With Walter Marshall, in 1971 he published Theory of Thermal Neutron Scattering, which has been an essential reference book for neutron scatterers, particularly in the field of magnetism, and which was influential in the development of the use of correlation functions for describing the scattering.

The present two volumes extend the coverage of the field beyond that of the earlier book; Vol. 1 describes elastic nuclear scattering, scattering by lattice vibrations and by liquids, and some chemical applications, while Vol. 2 deals with elastic and inelastic magnetic scattering. Although the second volume is entirely rewritten, most of its contents and the manner of treatment will be familiar to

\section{Advertisement}

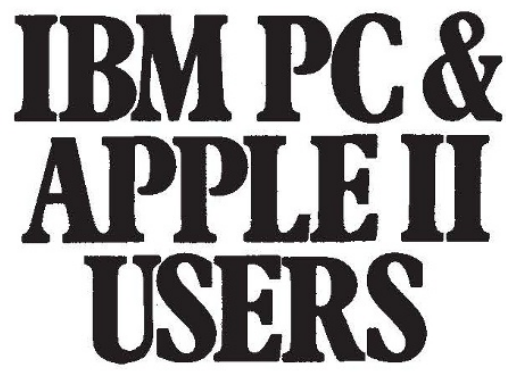

Dramatic advances are being made in scientific tools for personal computers. Jandel Scientific specializes in software and peripherals for:

- Planimetry, digitizing

- Interactive Statistics

- Dynamic System Simulation

- Computer-generated lecture Slides

- And more. .

Call us and let our technical advisors help you find the product you need. Or write or call for our FREE catalog.

\section{JANDEL SCIENTIFIC}

2656 BRIDGEWAY

SAUSALITO, CA 94965

CALL TOLL-FREE 800-874-1888

IN CALIF. CALL 415-331-3022

Reader Service No.02 readers of Theory of Thermal Neutron Scattering.

In neutron scattering, the Van Hove correlation function is a natural bridge between experiment and theory. The task of the experimentalist is frequently to perform measurements of the function which can then be interpreted in terms of a model for the particular system. Unfortunately there is very little in either of the books about the theory of how to obtain it from measurements of the scattered intensity. There is also nothing on the theory of the experimental resolution or other experimental constraints. The books are a helpful source for the calculation of the Van Hove function for a wide variety of soluble or nearly soluble models, and although I found them difficult to read

\section{Masters of swing}

\section{Russell H. Tuttle}

The Lesser Apes: Evolutionary and Behavioural Biology.

Edited by Holger Preuschoft et al: Edinburgh University Press/Columbia University Press: 1984. Pp. 709. £65, \$56.

DESPITE their modest size and colloquial label, the lesser apes (Hylobatidae) can claim a fair share of superlatives. They are the most numerous and variegated, relatively longest limbed, "swingingest", most naturally bipedal, most musical and most socially cohesive of the apes. We do not know whence they came or how they acquired their remarkable adaptive complexes; but few scientists would deny that we are blessed to have them grace the Earth.

Preuschoft and his colleagues have paid them just homage in this well-produced, fact-packed volume, which presents the augmented results of a conference held near Ulm, West Germany, in 1980. It will be a long-lasting source book on Hylobates. The organization of the work reflects its major purpose, that is to impress the world with the need to conserve forest habitats and thereby preserve the glorious gibbons of south-eastern Asia. Hence the first of the five sections is devoted to conservation. Its authors are soberly persuasive.

The plights of Javan silvery gibbons, Mentawai Kloss gibbons, South Asian hoolocks, Thai pileated gibbons and Chinese concolor gibbons are particularly acute. Our knowledge about the effects of high-technology warfare on wildlife in Kampuchea, Laos and Vietnam ranges from scanty to nil, but if the destruction of human life is any index the hylobatids probably have only a poor chance of long-term survival there.

It is now virtually certain that monogamy and territoriality are universal among the Hylobatidae, though we cannot yet tell () 1985 Nature Publishing Group they will undoubtedly prove valuable for detailed derivations of particular models. It is, however, a pity that the importance of the formulae produced are not illustrated through more examples of experimental results and discussion of when the simple models apply or do not apply to real systems.

Stephen Lovesey has again provided us with a very useful work of reference. These two volumes should not be used as textbooks because of the neglect of experimental aspects of the subject and of applications to real systems. The books, however, will be consulted by neutron scatterers for many years.

R.A. Cowley is Professor of Physics at the University of Edinburgh.

whether new groups are formed by the same mechanisms or whether defence of a section of the range serves comparable adaptive functions in all species. All of them call dramatically (and beautifully) but there is notable interspecific variation in which of the sexes calls, displays athletically, and chases and attacks in reaction to the calls of neighbours, actual intrusions or other factors. The interplay between calling, monogamy, subsistence and territorial defence in Hylobates has proved an attractive problem for a host of biologists, and Sections 3 and 4 of The Lesser Apes contain a wealth of information on the topic. Given their relevance to salient problems in theoretical biology, anthropologists can no longer be viewed as the proprietors of the hylobatid apes.

Functional morphologists have made great strides in elucidating the mechanisms underpinning hylobatid brachiation (armswinging), bipedalism and vertical climbing. As with conservation and ecological and behavioural puzzles, the lesser apes have ecumenically challenged Asian, European and North American anatomists and kinesiologists to explain their peculiar positional behaviour. Section 2 of the book is an excellent report of results from modern, technically and mathematically sophisticated, studies on hylobatid subjects and specimens. Here we learn that because of their elongated forelimbs, the movements of slowly brachiating hylobatids correspond reasonably well with the model of a mathematical pendulum, and that the elongation of the forelimbs economizes energy during rapid (ricochetal) arm-swinging.

It is heartening to see a reiteration (on pp. 94-95) of my 1975 model for the stem hominoids as hylobatid-sized, versatile arboreal beasts that were not especially adapted for dramatic brachiation. But I would have been happier had Herr Hollihn cited Tuttle among the long list of references that inspired him to adopt it. $\square$ at the University of Chicago. 